\title{
FRECUENCIA DE CARIES DENTALES EN PACIENTES DE LA CLÍNICA ODONTOLÓGICA DE LA UNIVERSIDAD TECNOLÓGICA CENTROAMERICANA
}

\author{
Michelle Cerrato, Daniel Canales, Bessy Guevara, Siryi Chirinos, Rubén \\ Ramírez, Thania Espinal ${ }^{1}$ \\ Facultad de Ciencias de la Salud, Universidad Tecnológica Centroamericana, \\ Tegucigalpa, Honduras
}

(Enviado: Marzo, 2019/Aceptado: Abril, 2019)

\begin{abstract}
Resumen
La caries dental es un proceso patológico complejo, de origen infeccioso y transmisible que afecta a las estructuras dentarias y se caracteriza por un desequilibrio bioquímico que de no ser revertido, conduce a cavitación y a alteraciones del complejo dentino-pulpar. Datos de la Organización Mundial de la Salud indican que la prevalencia de caries a nivel mundial es de $60-90 \%$ en escolares y casi el $100 \%$ en adultos. Aquí presentamos un estudio descriptivo realizado en la clínica odontológica de UNITEC entre Junio 2016 y Agosto 2017. De los 168 pacientes evaluados $95 \%$ presentó carie. Las piezas dentales más afectadas fueron las molares. La molar con más caries fue la 2.7 con 94 caries, seguida de la 1.6 con 92 caries, la 2.6 con 89 caries, la 3.7 con 88 caries y la 1.7 con 86 caries. El $78 \%$ de pacientes usa cepillo dental y solamente un $32 \%$ usa hilo dental. Es necesario promover más prevención dental.
\end{abstract}

Palabras Claves: Carie dental, estructura dental, diagnóstico, prevención y control, salud oral.

\begin{abstract}
Tooth decay is a complex pathological process of infectious origin that negatively affects dental structure. It is characterized by biochemical imbalances in dental tissues, if not treated can sause dental cavities and alterations in the dentine-pulp complex. Data by the World Health Organization indicate that prevalence ranges between 60$90 \%$ in young age people and almost $100 \%$ in adults. Here we present a descriptive, cross-sectional study conducted, between June 2016 and August 2017 at the dentistry clinic of UNITEC. Of the168 patients evaluated, $95 \%$ presented caries. The most affected teeth were molars. The molar with more caries was 2.7 with 94 caries, followed by 1.6 with 92 caries, 2.6 with 89 caries, 3.7 with 88 caries and 1.7 with 86 caries. Seventy-eight percent of patients use a toothbrush and only $32 \%$ use dental floss. It is necessary to promote more dental prevention.
\end{abstract}

Keywords: Dental caries, tooth decay, dental structure, prevention and control, oral health.

\footnotetext{
${ }^{1}$ Correspondencia: Dra. Thania Ramírez, email thania.espinal@unitec.edu.hn
} 


\section{Introducción}

La caries dental es una enfermedad ampliamente extendida en el mundo, ha sido y todavía sigue siendo la enfermedad crónica más frecuente que padece el ser humano. Muchos investigadores han propuesto diversas teorías sobre el origen de la caries dental. La caries dental así mismo puede definirse desde el punto de vista epidemiológico como una enfermedad que aparece en la infancia con el brote de los dientes ya que es la enfermedad bucal de mayor prevalencia y, en la mayor parte del mundo permanece como un problema sobresaliente de salud pública.

Según la Organización Mundial de la Salud (OMS), la caries es "Un proceso localizado de origen multifactorial que se inicia después de la erupción dentaria, determinando el reblandecimiento del tejido duro del diente y que evoluciona hasta la formación de una cavidad" (2012). Nuñez define la caries dental como un "proceso o enfermedad dinámica crónica, que ocurre en la estructura dentaria en contacto con los depósitos microbianos y, debido al desequilibrio entre la sustancia dental y el fluido de placa circundante, dando como resultado una pérdida de mineral de la superficie dental, cuyo signo es la destrucción localizada de tejidos duros" (Nuñez, 2010).

Existen varios factores causales de la caries dental, especialmente 4 factores generales: el huésped, el sustrato, la microflora y el tiempo (Núñez, 2010; González Sanz, 2013; Pereda \& González, 2014). El huésped va a proveer un ambiente favorable o desfavorable para la formación de la carie, ya sea por su nivel de higiene o por predisposición genética. El sustrato se refiere al tipo de dieta que tenga el huésped, en otras palabras, una dieta cariogénica o una cario-estática. Entre las bacterias que se relacionan con la formación de carie se encuentran: estreptococos sanguis, lactobacillus acidophilus y estreptococos mutans. Por último, se toma en cuenta el tiempo, debido a que los factores anteriormente mencionados deben estar en contacto por una cierta cantidad de tiempo para que el proceso cariogénico inicie.

Según la OMS y otros autores, la prevalencia de carie es de 60-90\% de los escolares y de 95 a $100 \%$ población general y mismo porcentaje entre personas adultas (OMS, 2012). Se considera que la prevalencia se ha reducido en países desarrollados (Hernandez et al., 2014). Siendo esta enfermedad cuya prevalencia es muy notoria, optamos por analizar una pequeña muestra de la clínica odontológica de la Universidad Tecnológica Centroamericana (UNITEC) en Tegucigalpa para establecer la frecuencia de caries en las personas que son atendidas y evaluadas en dicha clínica.

\section{Pacientes y Métodos}

Se realizó un estudio descriptivo, de corte transversal en la clínica odontológica de UNITEC. Se tomaron en cuenta los expedientes de pacientes que asistieron a la clínica entre las fechas de junio 2016 a agosto 2017. Entre los criterios de exclusión se mencionaba a los pacientes edéntulos (desdentados) y con dentición mixta.

Se realizó el cálculo de la muestra con un nivel de confianza del 95\%, precisión del 3\%, proporción del $5 \%$ y un ajuste por pérdidas del 15\%. Se llevó a cabo un muestreo probabilístico aleatorio simple resultando en una muestra de 168 expedientes. Se utilizó la plataforma virtual Smile de la clínica odontológica de la cual se obtuvieron los expedientes. Se aplicó un cuestionario con preguntas abiertas y cerradas de aspecto demográfico, patológico y medidas de prevención dental. 
- Se usó la Clasificación de Black así:

- Clase I: molar o premolar. Localizadas en la zona de fosas, surcos y fisuras.

- Clase II: dos o más superficies de una pieza. Suelen realizarse cuando existen caries proximales en molares y premolares.

- Clase III: Incisivos y caninos. Cuando hay una lesión que afecta a las superficies proximales de esas piezas y el borde incisal no está afectado.

- Clase IV: Incisivos y caninos, borde incisal. Cuando hay una lesión que afecta a las superficies proximales de esas piezas y el borde incisal está afectado.

- Clase V: Superficies lisas vestibulares o palatinas. Las más habituales: caries de cuello o abrasiones del esmalte.

- Clase VI. Este tipo no está recogido por Black. Hace referencia a lesiones que afectan a las cúspides de un diente posterior o a la superficie incisal de un diente anterior (caninos).

Los datos fueron analizados con el programa estadístico de Microsoft Excel 2010 y se hizo un análisis univariado, se utilizaron medidas de tendencia central como ser: promedio, moda y medidas de dispersión: rango y desviación estándar.

Se obtuvo la aprobación ética al elstudio por parte de las autoridades de la Facultad de Ciencias de la Salud. Se siguió las normas estándar de confidencialidad y protección de datos de los participantes. Se obtuvo consentimiento informado verbal.

\section{Resultados}

La muestra final estuvo constituida por 65\% (109/168) de pacientes femeninas y solo un 35\% (59/168) de pacientes masculinos. El porcentaje de pacientes con caries dentales que asisten a la clínica fue del 95\% (160/168). En la figura 1 se observa el grado de escolaridad de la muestra, que en su mayoría tiene un grado de escolaridad superior completa y superior incompleta.

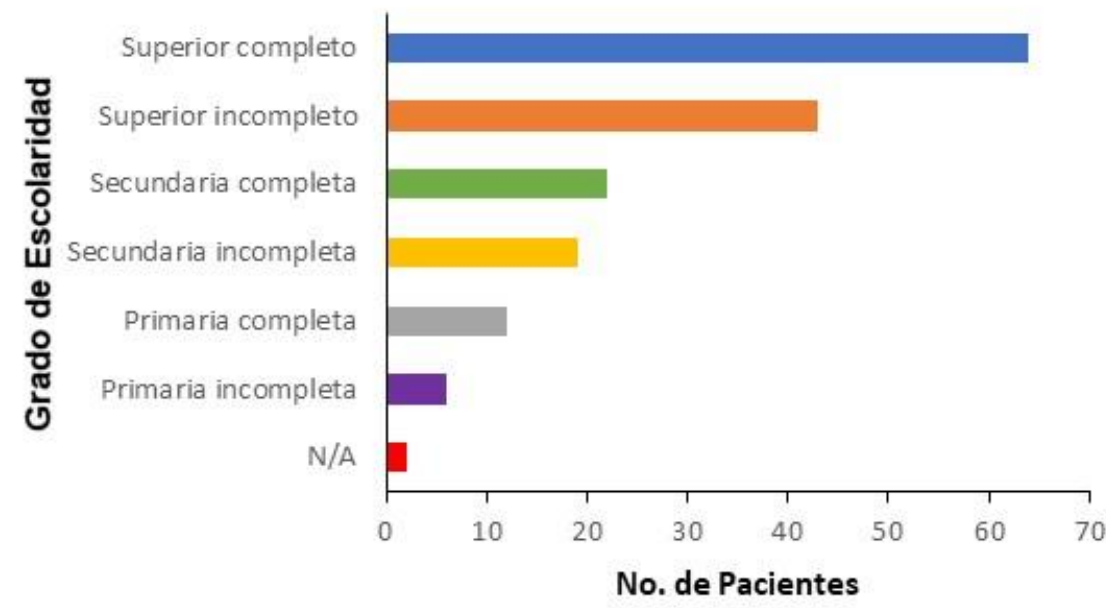

Figura 1. Grado de escolaridad de los pacientes evaluados en la clínica odontológica Fuente: elaboracion propia 
El figura 2 muestra la cantidad de caries por clase. La clase I con el mayor número de caries con un $84.53 \%(1,208 / 1,429)$ de caries seguida de la clase II que presento el $8.81 \%(126 / 1,429)$. El Gráfico 3 muestra que el grupo de dientes más afectado son los posteriores. Los dos dientes que presentaron mayor prevalencia de caries fueron 2.7 (94 caries) y 1.6 (92 caries). Los datos en color rojo representan el número de caries sumada de todos los pacientes según pieza dental. Los datos en color negro son la enumeración del órgano dental según la Federación Dental Internacional (FDI). Las piezas dentales que más presentaron caries fueron las molares. La molar con más caries fue la 2.7 con 94 caries, seguida de la 1.6 con 92 caries, la 2.6 con 89 caries, la 3.7 con 88 caries y la 1.7 con 86 caries.

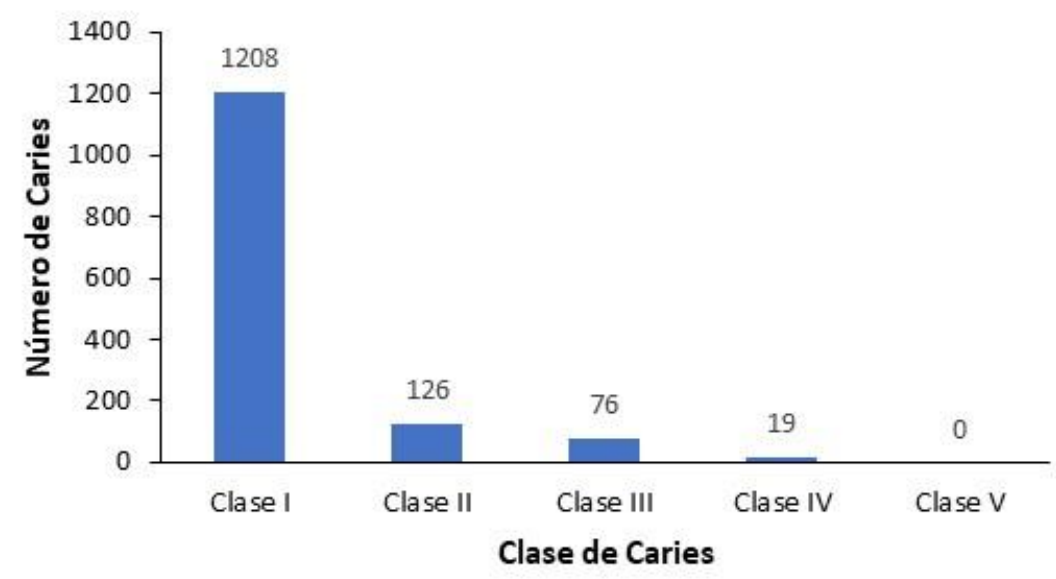

Figura 2. Cantidad de caries por clase según la clasificación de Black.

Fuente: elaboración propia

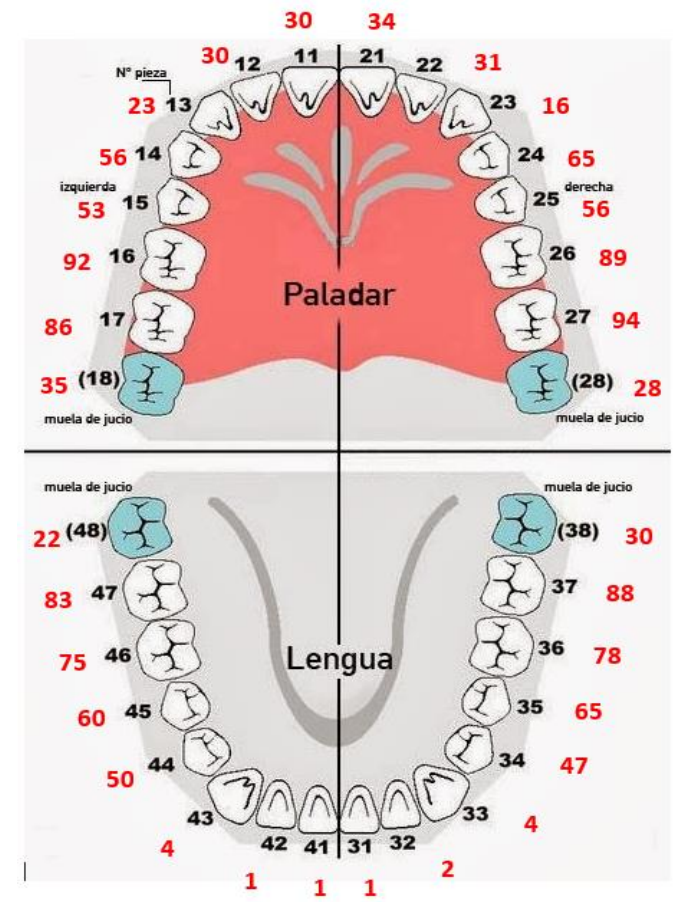

Figura 3. Número de caries que se presentan en piezas dentales de los pacientes evaluados.

Fuente: imagen recuperada de http://www.comparadentistas.com 
La Tabla 1 muestra que los pacientes entre las edades de 20 a 49 fueron los más afectados con caries. La Tabla 2 muestra que el $64 \%$ de las personas afectadas por caries dental son mujeres. Los resultados de la relación entre el grado de escolaridad y el número de caries están representados en la Tabla 3, que muestra que las personas más afectadas por caries son las que presentan un nivel de educación de superior completo. Las personas que tienen una escolaridad superior completa representan el 35\% (498/1429) de quienes tienen más caries, con un promedio de 7.78 caries por persona.

Tabla 1: Década de la vida en relación con el número de caries de los pacientes evaluados.

\begin{tabular}{llll}
\hline Edad & No. pacientes & No. caries & \% \\
\hline 6 a 10 & 3 & 1 & 0.07 \\
11 a 19 & 26 & 171 & 11.97 \\
\hline 20 a 29 & 49 & 459 & 32.12 \\
30 a 39 & 36 & 354 & 24.77 \\
40 a 49 & 35 & 311 & 21.76 \\
50 a 59 & 14 & 97 & 6.79 \\
60 a 69 & 4 & 36 & 2.52 \\
>de 70 & 1 & 0 & 0.00 \\
Total & $\mathbf{1 6 8}$ & $\mathbf{1 4 2 9}$ & $\mathbf{1 0 0 . 0 0}$ \\
\hline \multicolumn{4}{c}{ Fuente: elaboración propia }
\end{tabular}

Tabla 2: Número de caries relacionada con sexo. Se observó una relación hombre mujer 1.81.

\begin{tabular}{lrrr}
\hline Sexo & $\begin{array}{r}\text { No. de } \\
\text { Pacientes }\end{array}$ & $\begin{array}{c}\text { N. de } \\
\text { caries }\end{array}$ & $\%$ \\
\hline Femenino & 109 & 911 & $64 \%$ \\
Masculino & 59 & 518 & $36 \%$ \\
Total & $\mathbf{1 6 8}$ & $\mathbf{1 4 2 9}$ & $\mathbf{1 0 0 \%}$ \\
\hline
\end{tabular}

Fuente: elaboración propia

Tabla 3: Grado de escolaridad en relación al número de caries en los pacientes evaluados.

\begin{tabular}{lrrrr}
\hline $\begin{array}{l}\text { Grado de } \\
\text { Escolaridad }\end{array}$ & $\begin{array}{r}\text { No. de } \\
\text { Pacientes }\end{array}$ & \% & $\begin{array}{r}\text { No. de } \\
\text { Caries }\end{array}$ & \% \\
\hline N/C & 2 & 1 & 25 & 2 \\
Primaria incompleta & 6 & 4 & 27 & 2 \\
Primaria completa & 12 & 7 & 144 & 10 \\
Secundaria & & & & \\
incompleta & 19 & 11 & 142 & 10 \\
Secundaria completa & 22 & 13 & 241 & 17 \\
Superior incompleto & 43 & 26 & 352 & 25 \\
Superior completo & 64 & 38 & 498 & 35 \\
Total & $\mathbf{1 6 8}$ & $\mathbf{1 0 0}$ & $\mathbf{1 4 2 9}$ & $\mathbf{1 0 0}$ \\
\hline
\end{tabular}

Fuente: elaboración propia 
La frecuencia y la técnica de uso cepillo e hilo dental están directamente relacionadas con la prevalencia de caries. El 78\% de los pacientes que formaron parte de la muestra afirmaron si usar diariamente el cepillo dental, superando en gran medida a los que no lo usan (5\% de la población). El hilo dental en cambio, mostró resultados diferentes. Existe mayor cantidad de pacientes que no utilizan el hilo dental (50\%) a los que sí lo usan (32\%), ver Figuras 6 y 7.

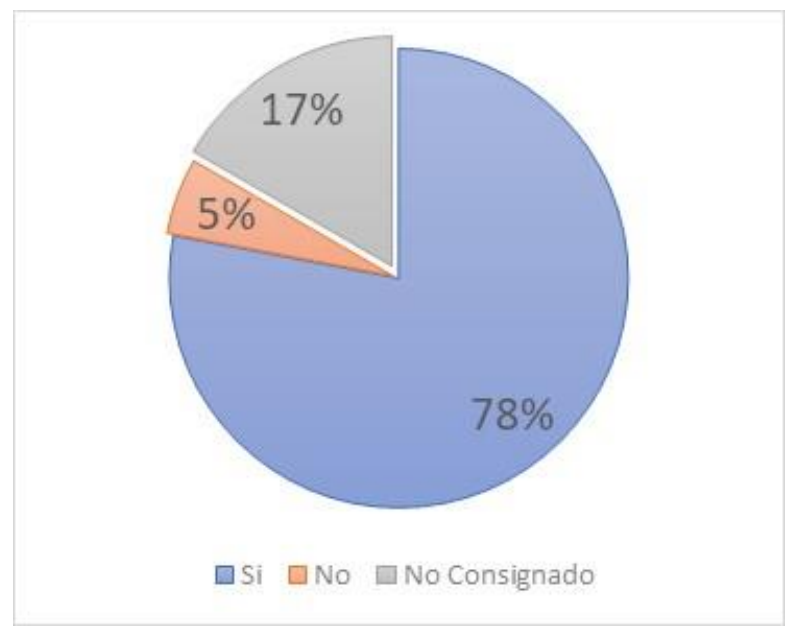

Figura 6: Porcentaje del uso de cepillo dental en pacientes entrevistados.

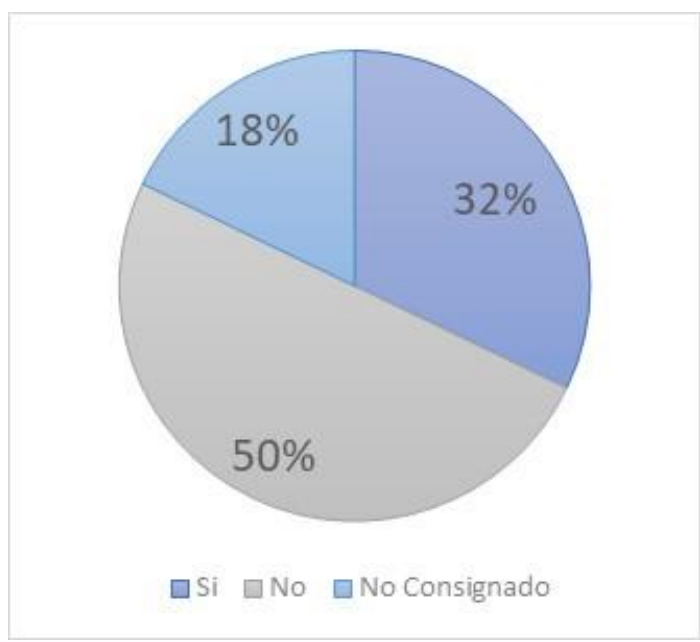

Figura 7: Porcentaje del uso de hilo dental en pacientes de la clínica odontológica de UNITEC.

\section{Discusion}

En este estudio se observó que el $95 \%$ de los pacientes presentaban caries, especialmente de Clase I y Clase II de la Clasificación de Black. Honduras siendo un país subdesarrollado, un factor influyente en la alta prevalencia de caries por varios factores, entre ellos la falta de educación y de atención dental a la población (OMS, 2012)

Un estudio sobre prevalencia de carie y enfermedad periodontal (EP) en estudiantes de odontología en la universidad de Cartagena, Colombia mostró una prevalencia de caries de 55.9\% (Arrieta, Caballero \& Martinez, 2012). Otro estudio realizado en jóvenes y adultos 
uruguayos del interior de ese país con una muestra de 922 participantes, dio como resultado un 94\% de prevalencia de caries en las mujeres y un 91\% en hombres (Olmos et al, 2013).

En Cuba la caries dental es una de las enfermedades bucales de mayor prevalencia, pues afecta a más del $90 \%$ de la población y la tendencia a padecerla en los menores de 15 años es un comportamiento similar al descrito por otros países. Se estima que la caries afecta aproximadamente al $40 \%$ de los infantes y entre el 60 y el $80 \%$ de los adolescentes y jóvenes (Hernandez et al., 2010).

La situación epidemiológica de la enfermedad no es muy diferente en Venezuela a la del resto de los países, ya que el $90 \%$ de la población venezolana menor de 13 años padecen la enfermedad. Estudios realizados en ese país en los últimos 25 años han mostrado que la prevalencia de la enfermedad entre los 10 y 12 años era de un $60 \%$, con tendencia a aumentar con la edad hasta alcanzar valores de 80-95 \% (Hernandez et al., 2013).

Un estudio por Arévalo y colaboradores, evaluó una muestra de 271 estudiantes de la Universidad Autónoma de Honduras. La evaluación de la muestra consistió en historia clínica, evaluación de la dieta y examen bucodental. Los resultados del estudio dieron un promedio de dientes con historia de caries dental de $14.6 \%$, sólo se encontraron 4 estudiantes universitarios libres de caries $(1.5 \%)$, el $72.1 \%$ tenía un índice de higiene oral aceptable y $27.9 \%$ no adecuado. $45.0 \%$ de los estudiantes presentaban periodontopatías (Arévalo, 2005).

En nuestro estudio, la clase de caries más común era la Clase I y II. Según Barrancos Mooney, la mayor prevalencia de caries clase I es debido al sistema de hoyos y fisuras que los órganos dentarios presentan en la cara oclusal y que comúnmente se extienden hacia las caras vestibular y palatina/lingual. Al igual él mismo justifica que las caries clase dos también son comunes debido al punto de contacto del diente, lo cual en condiciones anormales como ser de dientes con alteraciones en su posición pueden influir en el acumulo de residuos alimenticios y también albergar gran cantidad de bacterias (Mooney, 2015).

Nuestro hallazgo de la localización predominante de las caries puede ser debido al sistema de hoyos y fisuras presente en la cara oclusal dental. Otra razón que no se puede obviar es la dificultad de la higiene de estas piezas dentales debido a su posición en la arcada dentaria. Debido a que se encuentran bien posterior en la cavidad, las personas tienden a hacer un mal lavado y algunos casos más extremos ni siquiera les proporcionan lavado.

El hecho de que los pacientes entre las edades de 20-29 años estén más afectados por caries es diferente a los resultados presentados en estudios como el de Camagüey, Cuba, en el cual evaluaron a niños de entre 5 a 12 años. Los investigadores resaltaron el hecho de que en este rango de edad las personas eran especialmente susceptibles a las caries debido a su falta de habilidad manual y también a su falta de conocimiento en cuanto a las técnicas de cepillado. ${ }^{9}$ Otros autores indican que el paciente de sexo masculino tiene mayor riesgo de sufrir de caries debido a que pueden presentar un grado de higiene inferior que las pacientes de sexo femenino (Mooney, 2015).

Aunque la mayoría de los pacientes afirman utilizar cepillo dental, deberían tener una prevalencia de caries menor al 95\%. Queda inferir que la alta prevalencia de caries representada en nuestra investigación está relacionada a otros factores que no se tomaron en cuenta como por ejemplo técnica de cepillado inadecuada, consumo de tabaco, dieta altamente cariogénica, entre otros.

Debido a que los resultados demostraron una alta prevalencia de caries en los pacientes, consideramos esencial no solamente en tratar al paciente de la mejor manera posible, pero llevar el tratamiento desde un punto de vista restaurativo a un punto de vista preventivo. La educación 
del paciente es esencial para disminuir la prevalencia de carie ya que ellos son responsables de su propia higiene. Se recomienda a los estudiantes y a los directivos de la Clínica Odontológica de UNITEC incorporar y promover programas preventivos para nuestros pacientes.

\section{Referencias bibliográficas}

Organización Mundial de la Salud (OMS) [Internet]. Abril 2012 [Actualizado: 4/10/2018,

Citado: 8/6/2018]. OMS- Salud Bucodental, 1 página aproximada. Disponible en: http://www.who.int/mediacentre/factsheets/fs318/es/

Núñez DP, García BL. Bioquímica de la caries dental. Rev Haban Cienc Méd [Internet]. 2010 Jun [citado 2018 Dic 12] ; 9( 2 ): 156-166.

González Sanz AM, González Nieto BA, González Nieto E. Salud dental: relación entre la caries dental y el consumo de alimentos. Nutr. Hosp. [Internet]. 2013 Jul [citado 2018 Dic 13] ; 28( Suppl 4 ): 64-71.

Pereda Rojas ME, González Vera FE. Comportamiento del tabaquismo y la deficiente higiene bucal como factores de riesgo de la caries dental. CCM [Internet]. 2014 Dic [citado 2018 Dic 12] ; 18( 4 ): 623-635.

Mayor Hernández F, Pérez Quiñones JA, Cid Rodríguez MC, Martínez Brito I, Martínez Abreu J, Moure Ibarra MD. La caries dental y su interrelación con algunos factores sociales. Rev. Med. Electrón. [Internet]. 2014 Jun [citado 2018 Dic 12] ; 36( 3 ): 339-349.

Joseph R. The Father of Modern Dentistry - Dr. Greene Vardiman Black (1836-1915). J Conserv Dent 2005;8:5-6.

Arrieta Vergara KM, Díaz Caballero A, González Martínez F. Prevalencia de caries y enfermedad periodontal en estudiantes de odontología. Rev Cubana Estomatol [Internet]. 2011 Mar [citado 2018 Dic 12] ; 48( 1 ): 6-13.

Olmos Patricia PS, Musto Mariana LS, Álvarez R, Massa F. Caries dental. La enfermedad oral más prevalente: Primer estudio poblacional en jóvenes y adultos uruguayos del interior del país. Odontoestomatología [Internet]. 2013 Jun [citado 2018 Dic 13]; 15( spe ): 26-34.

Hernández Suárez A, Espeso Nápoles N, Reyes Obediente F, Rodríguez Borges L. Intervención educativo-curativa para la prevención de caries dental en niños de cinco a 12 años. AMC [Internet]. 2010 Dic [citado 2018 Dic 12] ; 14( 6 ): 1-9.

Hernández Marín CA, González Fortes B, Yero Mier I, Rivadeneira Obregón AM. Caries dental y la higiene bucal en adolescentes de 12 a 15 años. Área Norte Sancti Spíritus. 2010. Gac Méd Espirit [Internet]. 2013 Abr [citado 2018 Dic 12] ; 15( 1 ): 03-09. 
Arevalo SJ. (2005, 12 06). Situación de la Salud Bucal de la Población Universitaria Hondureña. Retrieved 06 06, 2017, from Rev. Med Honduras.

Mooney PB. (2015). Operatoria Dental. 5ta Ed. Editorial Médica Panamericana. 